\title{
Implications of the Intermediate Mass Black Hole in globular cluster G1 on Dark Matter detection
}

\author{
Gabrijela Zaharijaš \\ HEP Division, Argonne National Laboratory, 9700 Cass Ave., Argonne, IL 60439
}

\begin{abstract}
Recently there has been a growing evidence in favor of the presence of an Intermediate Mass Black Hole in the globular cluster G1, in Andromeda Galaxy. Under the assumption that formation of this globular cluster occurred within a dark matter halo, we explore whether the presence of a black hole could result in an observable gamma ray signal due to dark matter annihilation in this globular cluster. Starting from an initial NFW matter profile, with density parameters consistent with G1 observations, we find that indeed, if the spike in the density has been formed and has survived till present, the signal could be observed by GLAST and current ACT detectors.
\end{abstract}

PACS numbers: $95.35 .+\mathrm{d}$

ANL-HEP-PR-08-15

\section{INTRODUCTION}

One of the promising ways of detecting selfannihilating dark matter is through its final annihilation products, gamma rays being one of them. These, so called indirect detection techniques, offer an opportunity to identify dark matter (DM) properties, complementary to other searches, as dark matter direct detection and accelerator experiments. Gamma rays with energies higher than $1 \mathrm{GeV}$ have been searched for, in both satellite based (EGRET [1]) and ground-based experiments (HESS 2], MAGIC [3], CANGAROO [4], VERITAS [5],...) and new telescopes are being planned and built, such as GLAST [6] with launch scheduled for the next spring. The gamma ray signal of self-annihilating dark matter depends on the density squared and therefore "hot spots" where DM density is enhanced, are expected to exist across the sky. Promising sites for gamma ray DM detection should, therefore, have high DM density, be located in our vicinity and have a low level of astrophysical background, as the centers of our own and nearby galaxies, dwarf galaxies of the Milky Way, earth sized halos or primordial intermediate-mass black holes, for a review see [7].

In this paper we propose yet another site for dark matter indirect observation, the globular cluster G1 in the Andromeda Galaxy. This cluster has recently been observed with X-ray [8] and radio [9] telescopes and both measurements detected a source in the cluster core, consistent with the presence of an Intermediate Mass Black Hole (IMBH). Dynamical considerations, based on the most recent HST/WFPC2 and KECK/HIRES spectroscopic measurements support an IMBH presence, 10] (see also, 11, 12, 13]). In this letter we explore whether the presence of an IMBH could have an effect on the DM density favorable enough for G1 to be a promising target for indirect detection. Dark matter halos react to the growth of black holes. In the case of adiabatic growth of a central black hole, the density is strongly enhanced in its vicinity, resulting in a spike [14]. Since the gamma ray flux depends on the square of the density, a spike would enhance the flux by several orders of magnitude.
The existence and the slope of a spike is highly uncertain, however. We will later see how spikes with quite shallow slopes could still produce an observable signal.

G1 as a dark matter observation target suffers from being rather far from the Earth, but as we will see below, the enhancement of the density due to a black hole can potentially compensate for the distance. For example, if compared to the dwarf galaxy Draco, G1 is 10 times farther, but the signal from G1, in the case of a spike could be $10^{2 \div 4}$ times stronger than the one expected from Draco, 15]. Also, on the positive side, globular clusters are not expected to have significant background in highenergy gamma rays, as we will argue later. Relic IMBH have long been considered good sites for DM detection, but suffered from uncertainty in their location. If G1 really hosts an IMBH it would offer a unique opportunity to study these objects and their interaction with dark matter.

\section{GLOBULAR CLUSTERS AND G1}

Globular Clusters are very dense stellar systems: they have the typical mass of dwarf galaxies, but the size a factor of $\sim 10$ smaller. They are found in the halo or bulge regions of galaxies, [16]. Because they are old, they do not have significant amounts of interstellar gas and the stars within globular cluster are usually coeval. This suggests that the astrophysical background in high energy gamma ray telescopes should be small.

In the primordial scenario of globular cluster formation 17], globular clusters formed in DM minihaloes, before or during reionization, being the first galaxies to form; It is plausible, therefore, that globulars initially had a dominant dark matter component. They were then accreted by larger galaxies and lost their extra nuclear material due to stripping by the tidal forces. Today globular clusters are baryon dominated, with $M / L$ of a few. They have very dense stellar cores and the presence of tidal tails observed on globular cluster Palomar 5 suggests that they do not have an extended DM halo [18]. How much dark matter could be present in globular clusters? King- 
Michie models [19] do a satisfactory job in the dynamical description of globular clusters while considering only its stellar population. In that sense, presence of exotic dark matter is not required and no lower bound on DM annihilation signal from G1 can be placed. However, the simulations of Mashchenko and Sills [20] of the evolution of globular clusters in tidal fields show that the dark matter present in their nucleus could be compressed and its density profile steepened by these dynamical processes and that, depending on the initial dark matter profile, and type of DM-stellar interaction, some globular clusters could still present an extended halo.

The G1 cluster is one of the most luminous and the most massive globulars in the Local Group. Its total luminosity is $L \sim 210^{6} L_{\odot}$ and its mass is estimated to be between 1.4 and $1.7 \times 10^{7} M_{\odot}$ in King-Michie models, 21]. However, the values of the total mass of the cluster has large scatter depending on the model used for its calculation. For example Baumgardt et al. 13] find a value of $(8 \pm 1) 10^{6} M_{\odot}$ in their simulation which uses an evolutionary model. The main reason for these discrepancies is that spectroscopic data on G1 are rather poor: measurements of the velocity dispersion profile in the cluster was performed only in the inner region of the cluster (within $\sim 5 \mathrm{pc},[10]$ ) or the velocity dispersion was reduced to a single value, measured within a slit $\sim 6 \times 24 \mathrm{pc},[22]$.

G1 has a stellar core radius of $0.52 \mathrm{pc}$, and a half mass radius of $13 \mathrm{pc}$; its tidal radius is about $200 \mathrm{pc}, 21$. The cluster lies at a distance of $770 \mathrm{kpc}$ from us and it would be clearly separated from the bright Andromeda's disk in current high energy gamma ray telescopes.

G1 has several properties not typical for globular clusters. It has a very large central velocity dispersion [22], has a very flattened shape, with mean ellipticity of 0.2 and it shows a spread in metal abundances among its stellar population. In fact, one of the possible scenarios of G1 formation is that it is a surviving nucleus of a dwarf elliptical galaxy, which would have lost its outer envelop through tidal interaction with M31. In [23], Bekki and Chiba show that also in this scenario presence of DM in the core is plausible.

Evidence for a black hole in G1: While there is a growing knowledge of super-massive and stellar mass black holes, intermediate mass black holes are yet poorly understood. Recent detections of AGNs in low-luminosity, late-type galaxies suggest that IMBHs do exist (see, for instance, 24] ).

Several papers dealt with a dynamical analysis of the G1 cluster and the possible presence of an IMBH with different outcomes 10, 12, 13]. The most recent analysis by Gebhardt et al. [10] (G05), favors its presence. They find the best fit black hole mass to be $1.8 \pm 0.5 \times 10^{4} M_{\odot}$.

X-ray observations with XMM Newton reported a source with luminosity expected from an accretion onto an IMBH 25]. Radio observations, using Very Large Array detected a faint radio source within an arcsecond of the cluster core 99 with luminosity consistent with mass estimates by G05 and measured X-ray luminosity,

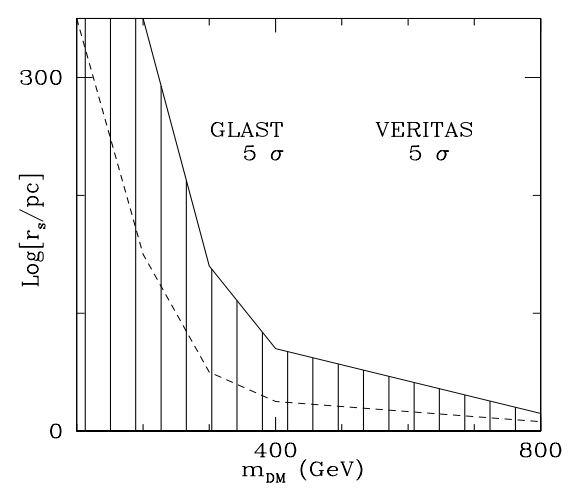

FIG. 1: The non-shaded region is the range of $r_{s}$ and $m_{D M}$ which could be detectable by GLAST or VERITAS with $5 \sigma$ significance or better. Shaded region is the excluded region from the EGRET observation. Solid line represents the case of $\Delta M=910^{4} M_{\odot}$, and dotted one $\Delta M=310^{4} M_{\odot}$.

thereby strengthening the evidence for a black hole presence.

\section{DARK MATTER DENSITY PROFILE}

We consider a cuspy NFW dark matter profile, $\rho(r)=$ $\rho_{0}\left(r / r_{s}\right)^{-1}\left(1+r / r_{s}\right)^{-2}$ where $\rho_{0}$ is central density and $r_{s}$ the scale radius.

Dark matter halos react to the growth of black holes, and in the case of adiabatic growth, the result is the formation of large over-densities called spikes, 14]. If the initial dark matter density was cuspy, after the adiabatic growth, the dark matter assumes a distribution given by

$$
\rho_{s p, N F W}(r)=\rho_{c u s p}\left(R_{s p}\right) g_{\gamma}(r)\left(\frac{R_{s p}}{r}\right)^{\gamma_{s p}},
$$

where $R_{s p}$ is the radius of influence of the black hole, found as a solution of the equation $\int_{0}^{R_{s p}} \rho(r) d^{3} r=2 M_{B H}$ $g_{\gamma}(r) \sim\left(1-4 R_{S} / r\right)^{3}$, where $R_{S}$ is the Schwarzchild radius of the black hole and $\gamma_{s p}=7 / 3$ in the NFW case.

On the other hand, in models with finite cores, the slope of the spike is shallower, $\gamma_{s p}=3 / 2$. Numerical simulations of halo formation predict cuspy profiles, 7]. However, simulations do not take into account the baryon-DM interactions and have other limitations, and we therefore consider a Burkert profile [26], which has a core at small $r, \rho(r)=\rho_{0}\left(1+r / r_{s}\right)^{-1}\left[1+\left(r / r_{s}\right)^{2}\right]^{-1}$. We parametrize the final dark matter profile after the growth of the spike as $\rho_{s p, c o r e}(r)=\rho_{0, \text { core }} g_{\gamma}(r)\left(R_{s p} / r\right)^{3 / 2}$.

Due to annihilations in the inner regions of the spike, there will exist a maximal dark matter density, given by $\rho_{\max }=m /\langle\sigma v\rangle_{B H}$, where $m$ is dark matter mass, $\langle\sigma v\rangle$ is the thermally averaged annihilation cross section and $t_{B H}$ is the age of IMBH, which we take to be $10^{10}$ years. The final dark matter profile is then given by $\rho_{s p, f}(r)=\rho_{s p} \rho_{\max } /\left(\rho_{s p}+\rho_{\max }\right)$. 
The adiabatic growth approach used here maximizes the effects of the black hole on the dark matter density profile. Substantial work has been done on a more realistic description of black hole influence on dark matter evolution, and the existence of a spike in our Galactic Center has been questioned, 27]. Various processes, such as offcenter black hole formation, black hole merging events, dark matter scattering by stars, etc. could all result in slopes shallower than predicted by the adiabatic growth model. The reconstruction of the central mass profile of G1, in G05, points to the existence of a cusp, both in the central luminosity and in the mass profiles. This in turn suggests that G1 did not go through a recent merger, which would deplete both stellar and dark matter cusps. On the other hand, stellar-dark matter scatterings might be important in G1. Gravitational scattering of dark matter with stars could deplete the spike and the slopes are generically well described with slope $3 / 2$, if this effects are taken into account, 28]. To probe this effect we somewhat artificially vary $\gamma_{s p}$ and find the lowest value which would result in a detectable signal.

To construct the DM density in G1 we start from the assumption that the outer part of the dark matter halo has been stripped together with the stellar envelope, while the dark matter in the center has survived due to the compactness of the core, as motivated by above mentioned simulations, [20, 23]. We conservatively truncate the profile at the tidal radius. Since the main contribution to the annihilation signal comes from the inner region of the profile $\left(r \lesssim r_{s}\right)$ this assumption would not significantly affect the result. Also, we conservatively assume that the inner slope of the density profile does not change with the evolution of the cluster.

The two parameters of the density distribution, $r_{s}$ and $\rho_{0}$, are poorly constrained in globular clusters. One typically constrains $r_{s}$ and $\rho_{0}$ by solving spherical Jeans equation, based on the velocity dispersion profile measurement, [29, 30]. In the case of G1, G05 find the mass density profile in the inner $5 \mathrm{pc}$, using this approach (the ellipticity of G1 is less than 0.1 in the innermost region of the cluster, 21], suggesting that the spherical approximation might be valid there), but determination of $r_{s}$ and $\rho_{0}$ is further complicated by the fact that G1 is baryon dominated, and that the stellar $M / L$ dependence on the radius is not well understood. We choose $r_{s}$ and $\rho_{0}$ by making sure that the dark matter density profile satisfies the three constraints listed below:

i) We use the uncertainty on the total cluster mass $\Delta M$ as the limit on the amount of dark matter allowed in the cluster: $\int \rho_{D M} d^{3} \vec{r}=\Delta M$. As $\Delta M$ we use both the error on the total mass within the King-Michie model $\left(310^{6} M_{\odot}\right)$ or the mass dispersion between various models $\left(910^{6} M_{\odot}\right)$.

ii) When adiabatic compression due to the black hole is present, the strongest limit might come from the measurement uncertainty of the total mass measured in the first bin $(\sim 0.1 \mathrm{pc})$, which is $\sim 310^{3} M_{\odot},[10$. We use this mass as an upper limit on the amount of DM in the central $\sim 0.1 \mathrm{pc}$

iii) We use the mass density profile, as found by G05, by imposing that the dark matter density should not be bigger than $10 \%$ of the found mass density of the cluster at any distance $(10 \%$ is somewhat arbitrary value, error bars on the mass density of the cluster as calculated in G05, are hard to estimate; the measurement error in the central bin is $15 \%$ ).

For comparison, we notice, that the values of $r_{s}$ (and $\rho_{0}$ ) can be estimated also by using the virial mass $M_{v i r}$ and the concentration parameter $c_{v i r}$, (for details, see [31]). If we take for $M_{v i r}$ to be $\sim 5$ times the mass of G1 today, it leads to a value of $r_{s}$ for the NFW profile of $\sim 270 \mathrm{pc}$, which is close to its tidal radius today.

\section{DETECTION SIGNAL}

In this section we estimate the strength of the dark matter annihilation signal as expected in the GLAST and ground-based Atmospheric Cerenkov Telescopes (ACTs). The EGRET experiment did not measure any signal above the diffuse gamma ray background in the direction of G1. We use this fact to place further limits on the value of $r_{s}$ and $\rho_{0}$.

In the case of G1, when the typical scale of the dark matter halo is much smaller than the distance to the object, the flux of gamma rays produced by self-annihilating dark matter is given by, [15],

$$
\Phi=\frac{N_{\gamma}}{4 \pi D^{2}} \int d r 4 \pi r^{2} \frac{\langle\sigma v\rangle}{2}\left(\frac{\rho(r)}{m_{d m}}\right)^{2}
$$

where $D$ is distance to the object and $m_{d m}$ is the dark matter mass; $N_{\gamma}$ is number of photons produced per annihilation and it is calculated here by means of the analytic fitting formula for the $\gamma$ ray spectrum produced by particles which annihilate dominantly to gauge bosons 32] which is a sufficiently accurate approximation for our purposes; we assumed a value for DM annihilation cross section $\langle\sigma v\rangle$ of $310^{-26} \mathrm{~cm}^{3} \mathrm{~s}^{-1}$, corresponding to the value expected for thermally decoupled relic.

For GLAST experiment we assume exposure of $1 \mathrm{~m}^{2} \mathrm{yr}$. The biggest contribution to the background for G1 comes from the Galactic diffuse emission at intermediate latitudes. Its strength is $\lesssim 610^{-6}(\mathrm{GeV} / \mathrm{E})^{-2} \mathrm{~cm}^{-2} \mathrm{~s}^{-1}$ $\mathrm{sr}^{-1} \mathrm{GeV}^{-1}$, 33]. Using field of view of $10^{-5} \mathrm{sr}$, this background accumulates to about 20 events in the given exposure.

For ACT experiments we assume an effective area of $410^{8} \mathrm{~cm}^{2}, 40$ hours of observation and an energy threshold of $100 \mathrm{GeV}$. The dominant background in ACTs comes from hadronic showers which are misidentified as gamma rays. Its spectrum is given as $d N_{\text {had }} / d E \sim$ $3 \epsilon_{\text {had }}\left(E / \mathrm{GeV}^{-2.7}\right) \mathrm{GeV}^{-1} \mathrm{sr}^{-1} \mathrm{~cm}^{-2} \mathrm{~s}^{-1}$, 15], where $\epsilon_{\text {had }}$ is the fraction of misidentified hadronic showers. For $\epsilon_{\text {had }}=0.01$, typical for ACTs, and a field of view of $10^{-5}$ sr, this background accumulates at a rate of $\sim 80$ events per hour of exposure. 
In the direction of G1, EGRET observed flux of $510^{-7} \mathrm{~cm}^{2} \mathrm{~s}^{-1} \mathrm{sr}{ }^{-1}$ above $1 \mathrm{GeV}$, 1]. The area of EGRET was $6400 \mathrm{~cm}^{2}$, the angular resolution $0.5^{\circ}$ and we will assume one year of effective time. The measured number of events, from the direction of G1, in the energy range $1-10 \mathrm{GeV}$ is $\sim 25$.

We show the results in a Figure 1, where a NFW profile is assumed. The lower shaded region is excluded by EGRET. The upper right corner of the figure is observable by either GLAST or VERITAS experiments, with $5 \sigma$ significance, or better. We did not consider values of $r_{s}$ higher than $300 \mathrm{pc}$, since the tidal radius of G1 is 200 pc. We comment here that spikes with slopes as shallow as $\gamma_{s p}=1.75$ could still be observable, albeit for a very small parameter space: say for $m=100 \mathrm{GeV}$, the observable range of $r_{s}$ would be $3 \mathrm{pc} \lesssim r_{s} \lesssim 5.2 \mathrm{pc}$ (for $\left.\Delta M=310^{6} M_{\odot}\right)$, but with the parameter space enlarging considerably for higher $\gamma_{s p}$. This is meant to illustrate that even slopes shallower than the ones predicted by adiabatic growth of a black hole, could potentially be observable. We did not perform a detailed analysis in this case, instead we varied ad hoc the values of $\gamma_{s p}$ in Eq. (1) in order to mimic complicated superposition of effects in the central DM region (initial steepening of DM cusp, motivated by [20], and various dynamical processes that might contribute to the depletion of the spike). If the dark matter profile contains no spike, we find that its selfannihilation signal would be unobservable by GLAST and current ACTs. We also find that the core profile would not produce an observable signal even if the adiabatic spike is present. The main reason for this is the combined effect of a shallow slope of a spike and strong constraints on DM density profile parameters in the case of core profile.

\section{SUMMARY}

In this paper we show that the dark matter annihilation signal from the globular cluster G1 in Andromeda could be within the range of GLAST and ACT experiments if the DM cusp has steepened due to the influence of an IMBH.

G1 suffers from a high uncertainty in the determination of its dark matter density profile; more measurements of velocity dispersion profile are needed, as well as a better understanding of the stellar mass-to-light ratio. G1 is also an extragalactic source, a factor of $\sim 10$ farther then typical dwarf galaxies of the Milky Way. On the positive sides, it has potentially an IMBH in its center which could substantially enhance the dark matter signal. Because of its old stellar population, G1 should not have a high astrophysical background in high energy gamma rays.

With the GLAST detector coming up in the next year many new gamma ray sources will be discovered. It is possible that some of them will be uniquely interpreted as a dark matter signal. If we are lucky, the signal from G1 could add some information in this respect and maybe help disentangling the dark matter-astrophysical properties.

The author would like to kindly thank Dan Hooper and Robyn Levine with whom the idea for the paper emerged and to Pasquale Serpico and Emiliano Sefusatti for many helpful comments. Work supported in part by the US Department of Energy, Division of High Energy Physics, under Contract DE-AC02-06CH11357.
[1] http://cossc.gsfc.nasa.gov/docs/cgro/cossc/egret/

[2] http://www.mpi-hd.mpg.de/hfm/HESS/HESS.html

[3] http://wwwmagic.mppmu.mpg.de/

[4] http://icrhp9.icrr.u-tokyo.ac.jp/index.html.

[5] http://veritas.sao.arizona.edu/.

[6] http://www-glast.stanford.edu/

[7] G. Bertone et al. Phys. Rept., 405:279-390, 2005.

[8] Albert K. H. Kong. 2007. astro-ph/0703662

[9] J. S. Ulvestad et al. 2007. arXiv:0704.1458 [astro-ph].

[10] K. Gebhardt et al. Astrophys. J., 634:1093-1102, 2005.

[11] K. Gebhardt et al. Astrophys. J., 578:L41-L46, 2002.

[12] H. Baumgardt et al. Astrophys. J., 582:L21-L24, 2003.

[13] H. Baumgardt et al. Astrophys. J., 589:L25-L28, 2003.

[14] P. Gondolo and J. Silk. Phys. Rev. Lett., 83:1719-1722, 1999.

[15] L. Bergstrom and D. Hooper. Phys. Rev., D73:063510, 2006.

[16] G. Meylan. Proceedings of the Second ICRA Network Workshop, Rome, Pescara, Italy, 1-5 February 1999.

[17] P. J. E. Peebles and R. H. Dicke. Astrophys. J., 154:891, 1968.
[18] B. Moore. Astrophys. J., 461:L13, 1996.

[19] J. E. Gunn and R. F. Griffin. Aj, 84:752-773, June 1979.

[20] S. Mashchenko and A. Sills. Astrophys. J., 619:258, 2005.

[21] G. Meylan et al. astro-ph/0105013.

[22] S. G. Djorgovski et al. apjl, 474:L19+, January 1997.

[23] K. Bekki and M. Chiba. Astron. Astrophys., 417:437-442, 2004.

[24] A. V. Filippenko and L. C. Ho. Astrophys. J., 588:L13L16, 2003.

[25] D. Pooley and S. Rappaport. Astrophys. J., 644:L45-L48, 2006.

[26] A. Burkert. IAU Symp., 171:175, 1996.

[27] M. Fornasa and G. Bertone. arXiv:0711.3148 [astro-ph].

[28] D. Merritt. Phys. Rev. Lett., 92:201304, 2004.

[29] S. Mashchenko et al. Astrophys. J., 640:252-269, 2006.

[30] L. E. Strigari et al. Phys. Rev., D75:083526, 2007.

[31] P. Ullio et al. Phys. Rev., D66:123502, 2002.

[32] L. Bergstrom et al. Astropart. Phys., 9:137-162, 1998.

[33] A. W. Strong et al. Astrophys. J., 613:962-976, 2004. 